\title{
Decline and repair, and covariate effects
}

\author{
Shaomin $\mathrm{Wu}^{1}$ \\ Kent Business School, University of Kent, \\ Canterbury, Kent CT2 7PE, United Kingdom \\ Philip Scarf \\ Salford Business School, Salford University, \\ Salford M5 $4 W T, U K$
}

\begin{abstract}
The failure processes of repairable systems may be impacted by operational and environmental stress factors. To accommodate such factors, reliability can be modelled using a multiplicative intensity function. In the proportional intensity model, the failure intensity is the product of the failure intensity function of the baseline system that quantifies intrinsic factors and a function of covariates that quantify extrinsic factors. The existing literature has extensively studied the failure processes of repairable systems using general repair concepts such as age-reduction when no covariate effects are considered. This paper investigates different approaches for modelling the failure and repair process of repairable systems in the presence of time-dependent covariates. We derive statistical properties of the failure processes for such systems.
\end{abstract}

Keywords: repair, proportional intensity model, virtual age, maintenance.

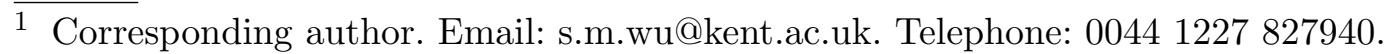




\section{Introduction}

\subsection{Motivation}

The failure process of systems in reliability theory may be described using a multiplicative intensity function that is the product of a baseline failure intensity function of the system and a function of time-dependent covariates. When repair modifies the argument (e.g. the virtual age of the system ${ }^{\text {ii }}$ ) of the baseline intensity function (for example, a repair can bring the system to a younger virtual age) but has no effect on the argument of the function of the time-dependent covariates, the argument in the baseline intensity function and the argument in the function of the time-dependent covariates become asynchronous. This leads to technical difficulties in estimating the probability of system failure at a given time point. This paper addresses this issue and studies the statistical properties of the failure process in this circumstance.

Knowledge of the effect of repair is important for determining optimum maintenance policy (Scarf, 1997; Dekker \& Scarf, 1998; Wang, 2002; Xiang, 2013; Zhong \& Jin, 2014; Kirschenmann, Popova, Damien, \& Hanson, 2014) and modelling the repair effect has attracted considerable research (e.g., Malik, 1979; Nakagawa, 1988; Kijima, 1989; Doyen \& Gaudoin, 2004; Guo, Liao, Zhao, \& Mettas, 2007; Wu \& Zuo, 2010). The repair effect can be accommodated by a number of means, such as modification of the failure intensity or reduction of the virtual age of the system. Readers are referred to Doyen and Gaudoin (2004) and Wu and Zuo (2010) for more detailed discussion of such maintenance models. In practice, the failure process of a system may be influenced by operational and environmental stress factors. Such influence can be accommodated by models such as:

$$
\lambda_{\mathrm{o}}(t)=\lambda_{\mathrm{b}}(t) \varphi(t)
$$

where $\lambda_{o}(t)$ is the failure intensity function of the system that is working in the field, in its operating environment, under the effect of extrinsic factors and that is referred to as the operational system hereafter, $\lambda_{\mathrm{b}}(t)$ is the baseline intensity function of the system - which is referred to as

ii The virtual age concept was introduced by Kijima (1989): suppose a system fails at calendar age $X$ since new and a repair of the system has the effect of modifying the system age to $X_{0}\left(X_{0}<X\right)$, then $X_{0}$ is called virtual age of the system. 
the baseline system in what follows - when the system is not subject to the effect of extrinsic factors, and $\varphi(t)$ is a function of covariates that quantify such extrinsic factors. When $\varphi(t)$ is an exponential function of the covariates, the model (1) is the proportional intensity model (e.g., Jiang, Landers, \& Rhoads, 2006).

When one models the failure process of repairable systems with time-dependent covariates, an issue arises: mathematically, do we consider that a repair affects $\lambda_{\mathrm{b}}(t)$ or that it affects $\lambda_{\mathrm{o}}(t)$ ? Consideration of this issue leads to one of the following three modelling assumptions.

Assumption A. Ignore extrinsic effects and model the failure probability and the repair effect only through $\lambda_{\mathrm{b}}(t)$.

Assumption B. Assume repair modifies the intensity function of the operational system in a synchronised manner so that in the case of an age reduction $\omega$ after a repair, $\lambda_{\mathrm{o}}(t)$ becomes $\lambda_{\mathrm{o}}(t-\omega)$ (where $\left.\omega>0\right)$, which implies that $\lambda_{\mathrm{o}}(t-\omega)=\lambda_{\mathrm{b}}(t-\omega) \varphi(t-\omega)$.

Assumption C. Assume repair only affects $\lambda_{\mathrm{b}}(t)$ while still accounting for the effect of extrinsic factors through the covariates.

While existing models of repair use the approach in Assumption A, this is not useful for modelling the reliability of systems subject to both intrinsic and extrinsic effects. Assumption B will accommodate extrinsic effects but it assumes that extrinsic factors are modified to the same degree of age reduction $\omega$ as the baseline system. However, in practical applications repair of the baseline system may not influence the covariate effects. As such, Assumption B may only be valid if $\varphi(t)$ can be modified to $\varphi(t-\omega)$ after repair. If the failure intensity function becomes $\lambda_{\mathrm{o}}(t-\omega)=\lambda_{\mathrm{b}}(t-\omega) \varphi(t-\omega)$ after repair, then one can simply analyse $\lambda_{\mathrm{o}}(t)$ as a whole and existing methods can be applied. With Assumption $\mathrm{C}$, the difficulty is that a repair may bring the baseline system to a younger stage, $\omega$ time units younger, say, but that this causes the following two problems. Firstly, the arguments in $\lambda_{\mathrm{b}}(t)$ and in $\varphi(t)$ will become asynchronous. That is, after a repair, $\lambda_{\mathrm{b}}(t)$ may become $\lambda_{\mathrm{b}}(t-\omega)$ but $\varphi(t)$ may remain the same (with $\varphi(t)$ unaffected by the repair). Secondly, the relationship $\lambda_{o}(t)=\lambda_{\mathrm{b}}(t-\omega) \varphi(t)$ may not hold.

This paper considers the failure process of repairable systems with time-dependent covariates under Assumption $\mathrm{C}$, and further assumes that $\lambda_{\mathrm{o}}(t)=\lambda_{\mathrm{b}}(t-\omega) \varphi(t)$ holds after repair. 


\subsection{Related work}

Starting with the work of Malik (1979), modelling the repair effect has been well studied in the literature. Published research to date includes the following methods.

Methods relating to the lifetime distribution function. This category models system survival times after each repair with a lifetime distribution function, $F_{n}(t)$, that changes with $n$, the number of repairs that the system has experienced. Examples include: the geometric process (Lam, 1988), in which $F_{n}(t)=F\left(a^{n-1} t\right)$, and where $a(>0)$ is an estimable parameter, and $F(t)$ is the lifetime distribution of the new system; and the extended Poisson process $\left(\mathrm{Wu} \&\right.$ Clements-Croome, 2006), in which $F_{n}(t)=F\left(\left(\alpha a^{n-1}+\beta b^{n-1}\right) t\right), \alpha, \beta, a$, and $b$ are estimable positive parameters.

Methods relating to the failure intensity function. This category assumes that repair modifies the failure intensity. For example, a model proposed in Wu and Zuo (2010) assumes that the failure intensity function of a system after the $n$th repair is linearly related to that before the $n$th repair. More detailed discussion of models in this category can be found in Nakagawa (1988), Doyen and Gaudoin (2004), and Wu and Zuo (2010).

Methods relating to the virtual age of the system. This category assumes that repair modifies the age of the system. For example, a model proposed in Kijima (1989) assumes that the virtual age of a system immediately after its $n$th repair is linearly related to that before the $n$th repair. More discussion of models in this category can be found in Nakagawa (1988), Kijima (1989), Doyen and Gaudoin (2004), and Wu and Zuo (2010).

While we have used the term repair in the above discussion, such repair may correspond to maintenance action carried out preventively (planned) or correctively (unplanned, following failure). In this paper the new results that we derive will however be concerned only with (unplanned) repair that follows failure. This is because the probability of system failure following planned preventive maintenance can be derived easily as the time such maintenance is conducted is pre-specified (e.g., Wu \& Zuo, 2010).

Many other models measuring the repair effect have been developed (see Wu \& Zuo, 2010) for a more detailed discussion). These models, however, do not consider scenarios in which the failure processes of systems are impacted by extrinsic factors quantified through covariates, especially time-dependent covariates, although Guo et al. (2007) considers a special scenario in 
which $\varphi(t)$ in equation (1) is assumed to follow a power law. When dealing with time-dependent covariates, one has to be very careful since there are some complications that arise with such scenarios. Kalbfleisch and Prentice (2002) point out that there are two types of time-dependent covariates: external and internal covariates. External time-dependent covariates are those whose values do not depend on the failure process. For example, when studying how long someone remains employed, the economic environment (quantified through e.g. the inflation rate) is essentially external to the individual's employment duration. Internal time-dependent covariates are often the measurements taken on the subjects. Since these time dependent measurements can only be taken when the subjects are under observation, the distribution of these covariates usually carries information on the failure process. Coming back to reliability theory and taking the reliability of a buried water pipeline as an example, external time-dependent covariates can quantify usage rates such as water pressure, and environmental stress factors such as soil properties and the pressure from the above ground, and internal time-dependent covariates can quantify the material properties of the pipe. In this paper, covariates are assumed to be external in the sense of Kalbfleisch and Prentice (2002).

\subsection{Contribution and importance of this work}

Derivation of the probability of failure for systems under Assumption A or Assumption B is established. The difficult case deals with Assumption C, in which the argument in the baseline intensity function and the argument in the function of the time-dependent covariates are asynchronous. It is this difficult case that we will consider in the paper and the development of theory for this case is the key contribution of our work.

The paper has important managerial implications. In asset management, planning maintenance regimes is one of the most important activities. This requires asset managers to understand the probability of failure of the assets in order to optimise maintenance intervals, to make their fiscal plans, etc. The models in this paper capture, in a better way than existing models, the effect of intervention upon the performance of a system that functions within a wider environment. The models therefore advance the state-of-the-art in intervention planning, and offer asset managers theoretically established methods that can be used in their projects.

Although this paper is developed using examples and terms such as repair and maintenance 
borrowed from reliability theory, its results and discussion can also be applied to any situations when recurrent events can be modelled with the multiplicative intensity function. Such applications can be found in scientific studies, medical research, marketing research, etc. In scientific studies each subject may repeatedly experience a certain phenomenon; medical examples of recurrent events are multiple infection episodes and tumour recurrences, and in marketing research examples of recurrent events include repeated purchases of a certain product. For example, Andersen and Gill (1982) built a proportional intensities model to describe admissions to psychiatric hospitals among women giving birth. One of the time-dependent covariates is the age of the women. It is obvious that for an individual patient, illness may respond to medical treatment but that such treatment could not change the age of a patient. In this example, models in which treatment (or, maintenance) does not influence covariates but does influence the baseline intensity of failure would seem appropriate.

\subsection{Overview}

The rest of the paper is structured as follows. Section 2, describes the failure intensity functions for different scenarios and lists definitions and assumptions. Section 3 offers a general discussion of possible methods of applying existing repair models to repairable systems with a timedependent covariates. Section 4 describes our main results for modelling the failure process of a repairable system under Assumption C. Section 5 presents a numerical illustration of our new results. We finish with a conclusion.

\section{Intensity functions}

This section defines the failure intensity functions for both operational and baseline systems. Note we assume no planned preventive maintenance is applied; repair is carried out on failure. When maintenance is planned the probability of system failure can be easily derived, as discussed earlier. We assume that maintenance times are negligible. Notation used in the paper is shown in Table 1. 
Table 1

Notation table

$\left\{N_{\mathrm{b}}(t)\right\}_{t \geq 0} \quad$ number of failures of the baseline system in $(0, t]$, a stochastic process

$\left\{N_{\mathrm{o}}(t)\right\}_{t \geq 0} \quad$ number of failures of the operational system in $(0, t]$, a stochastic process

$\mathscr{H}_{\mathrm{b}}(t)$ history of failures of the baseline system in $(0, t]$

$\mathscr{H}_{\mathrm{o}}(t)$ history of failures of the operational system in $(0, t]$

$V_{\mathrm{b}, n} \quad$ virtual age of the baseline system immediately after the $n$th repair

$V_{\mathrm{o}, n} \quad$ virtual age of the operational system immediately after the $n$th repair

$\lambda(t) \quad$ failure intensity of the new baseline system

$\lambda_{\mathrm{b}}(t)$ failure intensity of the baseline system

$\lambda_{\mathrm{o}}(t)$ failure intensity of the operational system

$z(t)$ a covariate, defined for $t \geq 0$; only one covariate is assumed

$\mathscr{Z}(t) \quad \mathscr{Z}(t)=\{z(\tau): \tau<t\}$, the covariate history

$\varphi($.$) a function$

$T_{\mathrm{b}, n} \quad$ successive failure times of the baseline system, starting from $T_{\mathrm{b}, 0}=0$

$T_{\mathrm{o}, n} \quad$ successive failure times of the operational system, starting from $T_{\mathrm{o}, 0}=0$

\subsection{Intensity functions for the baseline system}

The failure process of the baseline system is defined by the stochastic process $\left\{N_{\mathrm{b}}(t)\right\}_{t \geq 0}$ and characterised by the intensity function

$$
\lambda_{\mathrm{b}}(t)=\lim _{\Delta t \rightarrow 0} \frac{P\left\{N_{b}(t+\Delta t)-N_{\mathrm{b}}(t) \geq 1 \mid \mathscr{H}_{\mathrm{b}}(t)\right\}}{\Delta t}
$$

where $P\left\{N_{b}(t+\Delta t)-N_{\mathrm{b}}(t) \geq 1 \mid \mathscr{H}_{\mathrm{b}}(t)\right\}$ is the probability that the baseline system fails within the interval $(t, t+\Delta t)$, given the history of failures $\mathscr{H}_{\mathrm{b}}(t)$. Here, we will consider the situations in which the repair effect can be expressed either as a modification of failure intensity or as a modification of the virtual age.

- When the repair modifies the failure intensity, the failure intensity is given by

$$
\lambda_{\mathrm{b}}(t)=\mathscr{F}\left(\lambda(t), \lambda\left(T_{\mathrm{b}, 1}\right), \lambda\left(T_{\mathrm{b}, 2}\right), \ldots, \lambda\left(T_{\mathrm{b}, N_{\mathrm{b}}(t)}\right)\right)
$$

where $\mathscr{F}($.$) is a strictly positive and non-decreasing function. In the case N_{\mathrm{b}}(t)=0, \lambda_{\mathrm{b}}(t)=$ $\lambda(t)$. For example, if the repair effect is measured by the arithmetic reduction of intensity model $\left(\mathrm{ARI}_{1}\right)$ (see (Doyen \& Gaudoin, 2004) for details), we have

$$
\lambda_{\mathrm{b}}(t)=\lambda(t)-\rho_{0} \lambda\left(T_{\mathrm{b}, N_{\mathrm{b}}(t)}\right)
$$

and $\mathscr{F}\left(\lambda(t), \lambda\left(T_{\mathrm{b}, 1}\right), \lambda\left(T_{\mathrm{b}, 2}\right), \ldots, \lambda\left(T_{\mathrm{b}, N_{\mathrm{b}}(t)}\right)\right)=\lambda(t)-\rho_{0} \lambda\left(T_{\mathrm{b}, N_{\mathrm{b}}(t)}\right)$, where $\rho_{0} \in[0,1]$. 
- When the repair effect modifies the virtual age of the system, the failure intensity is given by

$$
\lambda_{\mathrm{b}}(t)=\lambda\left(\mathscr{G}\left(t, T_{\mathrm{b}, 1}, T_{\mathrm{b}, 2}, \ldots, T_{\mathrm{b}, N_{\mathrm{b}}(t)}\right)\right),
$$

where $\mathscr{G}($.$) is a strictly positive and non-decreasing function. For example, if the repair effect$ is measured by the arithmetic reduction of age model ( $\left.\mathrm{ARA}_{1}\right)$ (Doyen \& Gaudoin, 2004), we have

$$
\lambda_{\mathrm{b}}(t)=\lambda\left(t-\rho_{1} T_{\mathrm{b}, N_{\mathrm{b}}(t)}\right) .
$$

and $\mathscr{G}\left(t, T_{\mathrm{b}, 1}, T_{\mathrm{b}, 2}, \ldots, T_{\mathrm{b}, N_{\mathrm{b}}(t)}\right)=t-\rho_{1} T_{\mathrm{b}, N_{\mathrm{b}}(t)}$, where $\rho_{1} \in[0,1]$.

\subsection{Intensity function of the operational system}

The failure process of the operational system is defined by the stochastic process $\left\{N_{\mathrm{o}}(t)\right\}_{t \geq 0}$ and characterised by the intensity function

$$
\lambda_{\mathrm{o}}(t)=\lim _{\Delta t \rightarrow 0} \frac{P\left\{N_{o}(t+\Delta t)-N_{\mathrm{o}}(t) \geq 1 \mid \mathscr{H}_{\mathrm{o}}(t), \mathscr{Z}(t)\right\}}{\Delta t} .
$$

As we can see $\lambda_{\mathrm{b}}(t)$ defined in Eq. (2) is not impacted by the covariate history $\mathscr{Z}(t)$ but $\lambda_{\mathrm{o}}(t)$ defined in Eq. (7) is impacted by the covariate history. This creates a technical difficulty in the calculation of the probability of failure following repair that we discuss now.

\section{The effect of repair in the presence of a covariate}

When considering the repair effect on a system modelled with Eq. (1), one may make one of the three assumptions: Assumptions A, B, and C. Let us re-discuss these three assumptions below in the context of virtual age reduction at repair.

Assumption A. Here covariate(s) is (are) ignored so that $\lambda_{\mathrm{o}}(t)=\lambda_{\mathrm{b}}(t)$ and repair effects are captured in equations (3) and (5).

Assumption B. After repair, $\lambda_{\mathrm{o}}(t)$ becomes $\lambda_{\mathrm{o}}(t-w)$. That is, the repair takes effect on both the baseline system and the covariate. Thus, both $\lambda_{\mathrm{b}}(t)$ and $z(t)$ are influenced by maintenance activities to the same extent. Under this assumption, one can derive the following result:

$$
\lambda_{\mathrm{o}}(t)=\mathscr{M}\left(\lambda_{\mathrm{b}}(t), \varphi(t)\right),
$$


where $\mathscr{M}\left(\lambda_{\mathrm{b}}(t), \varphi(t)\right)$ is a synchronized form of $\varphi(t)$ with $\lambda_{\mathrm{b}}(t)$ with respect to both time and functional form. For example, under Assumption B and repair effect shown in Eq.(4), $\lambda_{\mathrm{o}}(t)$ becomes

$$
\begin{aligned}
\lambda_{\mathrm{o}}(t) & =\mathscr{F}\left(\mathscr{M}\left(\lambda_{\mathrm{b}}(t), \varphi(t)\right), \mathscr{M}\left(\lambda_{\mathrm{b}}\left(T_{\mathrm{o}, 1}\right), \varphi\left(T_{\mathrm{o}, 1}\right)\right), \ldots, \mathscr{M}\left(\lambda_{\mathrm{b}}\left(T_{\mathrm{o}, N_{\mathrm{o}}(t)}\right), \varphi\left(T_{\mathrm{o}, N_{\mathrm{o}}(t)}\right)\right)\right. \\
& =\lambda(t) \varphi(t)-\rho_{0} \lambda\left(T_{\mathrm{o}, N_{\mathrm{o}}(t)}\right) \varphi\left(T_{\mathrm{o}, N_{\mathrm{o}}(t)}\right) .
\end{aligned}
$$

Under Assumption B and repair effect shown in Eq.(6), $\lambda_{\mathrm{o}}(t)$ becomes

$$
\begin{aligned}
\lambda_{\mathrm{o}}(t) & =\mathscr{M}\left(\lambda_{\mathrm{b}}\left(\mathscr{G}\left(t, T_{\mathrm{o}, 1}, T_{\mathrm{o}, 2}, \ldots, T_{\mathrm{o}, N_{\mathrm{o}}(t)}\right)\right), \varphi\left(\mathscr{G}\left(t, T_{\mathrm{o}, 1}, T_{\mathrm{o}, 2}, \ldots, T_{\mathrm{o}, N_{\mathrm{o}}(t)}\right)\right)\right) \\
& =\lambda\left(t-\rho_{1} T_{\mathrm{o}, N_{\mathrm{o}}(t)}\right) \varphi\left(t-\rho_{1} T_{\mathrm{o}, N_{\mathrm{o}}(t)}\right) .
\end{aligned}
$$

Eqs. (9) and (10) essentially imply that repair affects both the operational system and the covariate to the same extent. As can be seen from Eqs. (9) and (10), $\varphi(t)$ changes after a repair.

Assumption C. Here repair affects $\lambda_{\mathrm{b}}(t)$ and not $\varphi(t)$, but we still wish to take account of extrinsic factors through a covariate. We now discuss the main focus of this paper: investigating the failure process of repairable systems with time-dependent covariates under this assumption.

\section{Modelling the failure process under Assumption C}

In this section, we derive the probability of system failure at time $t$ under Assumption C.

\section{$4.1 \varphi(t)=\varphi_{0}\left(\varphi_{0}\right.$ is constant $)$}

When $\varphi(t)=\varphi_{0}$, the covariates are time-independent. In this case, Eq. (1) becomes

$$
\lambda_{\mathrm{o}}(t)=\varphi_{0} \lambda_{\mathrm{b}}(t)
$$

For both age reduction models and intensity modification models, determining the probability of system failure at time $t$ proceeds as in Section 2.1. 


\subsection{When the repair effect is modification of the failure intensity}

In this case, Eq. (3) becomes $\lambda_{\mathrm{o}}(t)=\mathscr{F}\left(\lambda(t), \lambda\left(T_{\mathrm{o}, 1}\right), \lambda\left(T_{\mathrm{o}, 2}\right), \ldots, \lambda\left(T_{\mathrm{o}, N_{\mathrm{o}}(t)}\right)\right) \varphi(t)$. This is because repair simply modifies the intensity function, so that the arguments of the intensity function of the baseline system and the covariate remain untransformed and they remain synchronised, and determining the probability of system failure at time $t$ is in principle straightforward. This implies that for the $\mathrm{ARI}_{1}$ model Eq. (4) becomes $\lambda_{\mathrm{b}}(t)=\lambda(t) \varphi(t)-\rho_{0} \lambda\left(T_{\mathrm{o}, N_{\mathrm{o}}(t)}\right) \varphi(t)$.

\subsection{When the repair effect is modification of age}

Now, determining the probability of system failure at time $t$ becomes more difficult. In this subsection, we will consider the probability of system failure when age reduction in the baseline intensity is according to the $\mathrm{ARA}_{1}$ model. The age reduction model $\mathrm{ARA}_{1}$ shown in Eq. (6) can be derived from one of the virtual age models (Kijima, 1989).

Let $\rho_{n}$ denote the degree of the $n$th repair effect, where $\rho_{n} \in[0,1]$ (where $n=1,2, \ldots$ ), $V_{\mathrm{b}, n}$ the virtual age of the baseline system immediately after the $n$th repair (where $V_{\mathrm{b}, 0}=0$ ), and $X_{\mathrm{b}, n}$ the time between the $(n-1)$ th and the $n$th repair of the baseline system. Then the $\mathrm{ARA}_{1}$ model implies,

$$
V_{\mathrm{b}, n}=V_{\mathrm{b}, n-1}+\rho_{n} X_{\mathrm{b}, n}
$$

Here $\rho_{n} X_{\mathrm{b}, n}$ is the virtual age increase of the baseline system between the $(n-1)$ th and the $n$th repair.

If Eq. (12) is assumed, then a repair takes effect on $\lambda_{\mathrm{b}}(t)$, but not $\varphi(t)$. This causes a problem on Eq. (1), as explained in the following.

(a) After a repair on the baseline system, $\lambda_{\mathrm{b}}(t)$ can be changed. The change may follow, for example, the pattern shown in Eq. (3) or that shown in Eq. (5). $\varphi(t)$, however, may stay unchanged. This can lead to the following two scenarios:

(a.1) Model (1) may not hold any more; and

(a.2) Model (1) still holds.

(b) In case one assumes (a.1), deriving the probability of system failure becomes impossible, as the function $\lambda_{\mathrm{o}}(t)$ for some time $t$ following repair cannot be derived. 
(c) If the above scenario (a.2) holds, then the following issue arises: the concept of the virtual age can only be used for a system with a given form of $\lambda_{o}(t)$ for the operational system. We hence need to assume that $\lambda_{\mathrm{b}}(t)$ and $\lambda_{\mathrm{o}}(t)$ share the same form, or they are conjugate failure intensity functions. The term conjugate is borrowed from the concept of the conjugate distributions used in Bayesian statistics. The virtual age of the operational system immediately following the $n$th repair is then,

$$
V_{\mathrm{o}, n}=V_{\mathrm{o}, n-1}+X_{\mathrm{o}, n}^{\prime}
$$

where $V_{\mathrm{o}, 0}=0$, and $X_{\mathrm{o}, n}^{\prime}$ is the virtual age increase of the operational system between the $(n-1)$ th and the $n$th repairs.

Let $X_{\mathrm{o}, n}$ denote the time between the $(n-1)$ th and the $n$th repair of the operational system. Unlike model (12) in which the virtual age increase between repairs equals $\rho_{n} X_{\mathrm{b}, n}$, deriving an explicit relationship between $X_{\mathrm{o}, n}^{\prime}$ and $X_{\mathrm{o}, n}$ is not possible as the level of the repair effect on the operational system is unknown. It should also be noted that $V_{\mathrm{b}, n}$ and $V_{\mathrm{o}, n}$ are different as $V_{\mathrm{o}, n}$ is influenced by the covariate $z(t)$. Thus, deriving the failure process becomes more complicated if the model in Eq. (13) is used. This is because repair modifies the argument of the intensity function, which results in the fact that the virtual age of the operational system, i.e., the argument in the intensity function, differs from (or smaller than) the argument of the covariate. Below we resolve this issue.

Let $\bar{F}_{\mathrm{b}, i}(t)$ and $\bar{F}_{\mathrm{o}, i}(t)$ be the survivor functions after the $i$ th repair of the baseline system and the operational system, respectively, where $i=1,2, \ldots, \bar{F}_{\mathrm{b}, i}(t)=1-F_{\mathrm{b}, i}(t)$, and $\bar{F}_{\mathrm{o}, i}(t)=1-F_{\mathrm{o}, i}(t)$. $\bar{F}_{\mathrm{b}, 0}(t)$ and $\bar{F}_{\mathrm{o}, 0}(t)$ are the survivor functions of the baseline system and the operational system prior to the first failure, respectively. Then one can easily obtain $\bar{F}_{\mathrm{b}, 0}(t)=\exp \left\{-\int_{0}^{t} \lambda_{\mathrm{b}}(u) d u\right\}$ and $\bar{F}_{\mathrm{o}, 0}(t)=\exp \left\{-\int_{0}^{t} \lambda_{\mathrm{o}}(u) d u\right\}$. It should be noted that $\lambda_{\mathrm{b}}(t)$ is specified whereas $\lambda_{\mathrm{o}}(t)$ is unknown.

Let $\rho_{n}=\rho$ in the model shown in Eq. (12), where $\rho>0$. It then follows that

$$
V_{\mathrm{b}, n}=\rho \sum_{i=1}^{n} X_{\mathrm{b}, i} .
$$

where $\sum_{i=1}^{n} X_{\mathrm{b}, i}=T_{\mathrm{o}, N_{\mathrm{o}}(t)}$.

Denote $y$ by the virtual age of the baseline system, according to (Kijima, 1989), the survivor 
function of $V_{\mathrm{b}, n}$, denoted by $\bar{G}_{\mathrm{b}, n}(x)$, is given by

$$
\bar{G}_{\mathrm{b}, n}(x)=\bar{G}_{\mathrm{b}, n-1}(x)+\int_{0}^{x} \frac{\bar{F}_{\mathrm{b}, 0}\left(y+\frac{x-y}{\rho}\right)}{\bar{F}_{\mathrm{b}, 0}(y)} d G_{\mathrm{b}, n-1}(y) .
$$

where $G_{\mathrm{b}, n-1}(y) .=1-\bar{G}_{\mathrm{b}, n-1}(y)$ and $x>0$.

\subsubsection{General case}

In this subsection, we assume that the form of $z(t)$ is unknown.

Let $\mathscr{E}=\{$ the event that the covariate is not influenced by a repair conducted on the baseline system\}. $\mathscr{E}$ implies: when the baseline system approaches the virtual age $y$, the age (or calendar time) of the covariate is $y / \rho$.

Then we have the following Lemma.

Lemma 1 The probability that the operational system fails after the $n$th repair if the virtual age before the repair is $y$, is given by

$$
\operatorname{Pr}\left\{X_{\mathrm{o}, n} \leq x \mid V_{\mathrm{o}, n-1} \geq y\right\}=1-\exp \left\{-\int_{y}^{x+y} \lambda_{\mathrm{b}}(u) \varphi\left(u+\frac{(1-\rho) y}{\rho}\right) d u\right\}
$$

The proofs of Lemma 1 and the other lemmas and theorems can be found in the Appendix.

Denote the distribution of $V_{\mathrm{o}, n}$ by $G_{\mathrm{o}, n}(x)$. Then we have the following lemma.

Lemma 2 For model (13), one obtains

$$
\begin{aligned}
\bar{G}_{\mathrm{o}, n}(x) & =1-G_{\mathrm{o}, n}(x) \\
& =\bar{G}_{\mathrm{o}, n-1}(x)+\int_{0}^{x} \exp \left\{-\int_{y}^{y+\frac{x-y}{\rho}} \lambda_{\mathrm{b}}(u) \varphi\left(u+\frac{(1-\rho) y}{\rho}\right) d u\right\} d G_{\mathrm{o}, n-1}(y) .
\end{aligned}
$$

Let

$$
g_{x, \rho}(y)= \begin{cases}\exp \left\{-\int_{y}^{y+\frac{x-y}{\rho}} \lambda_{\mathrm{b}}(u) \varphi\left(u+\frac{(1-\rho) y}{\rho}\right) d u\right\}, & \text { if } y \leq x \\ 1, & \text { if } y>x\end{cases}
$$

Then, Eq. (17) can be re-written as

$$
\bar{G}_{\mathrm{o}, n}(x)=\int_{0}^{\infty} g_{x, \rho}(y) d G_{\mathrm{o}, n-1}(y)
$$

Lemma 3 If both $\varphi(u)$ and $\lambda_{b}(u)$ are non-decreasing, $g_{x, \rho}(y)$ is increasing with respect to $\rho$. 
Let $A_{1}, A_{2}, \ldots$ and $B_{1}, B_{2}, \ldots$ denote random sequences of the repair effect $\rho_{1}, \rho_{2}, \ldots$ at repairs $1,2, \ldots$ Further let $S_{\mathrm{o}, n}^{A}=\sum_{i=1}^{n} X_{\mathrm{o}, i} \mid\left\{\rho_{1}=A_{1}, \rho_{2}=A_{2}, \ldots, \rho_{n}=A_{n}\right\}$ and $S_{\mathrm{o}, n}^{B}=$ $\sum_{i=1}^{n} X_{\mathrm{o}, i} \mid\left\{\rho_{1}=B_{1}, \rho_{2}=B_{2}, \ldots, \rho_{n}=B_{n}\right\}$, that is, conditional on the degree of the $i$ th repair effect being $A_{i}$, and $B_{i}$ respectively, $i=1,2, \ldots, n$.

For non-negative random variables $X$ and $Y$, we denote $X \prec_{d} Y$ if $E[f(X)] \leq E[f(Y)]$ for any increasing function $f$. The ordering $\prec_{d}$ is called the stochastic ordering. Then we have the following theorem.

Theorem 1 If both $\varphi(u)$ and $\lambda_{b}(u)$ are non-decreasing and $A \prec_{d} B$, then $E\left[S_{\mathrm{o}, n}^{A}\right] \geq E\left[S_{\mathrm{o}, n}^{B}\right]$ for all $n \geq 1$.

The assumption $A \prec_{d} B$ implies that the sequence $A$ possesses a better maintenance effect than the sequence $B$.

\subsubsection{A special case}

$\varphi(t)$ in model (1) can take different forms. For example, in the case that model (1) is the proportional intensity model, $\varphi(t)=\exp \{\beta z(t)\}$, the value of $z(t)$ can be assumed to be constant in an interval but varying from one interval to the other. The approaches of Meyer (1990) and Han and Hausman (1990) follow this strategy. That is,

$$
\varphi(t)=\sum_{i=0}^{m} v_{i} I_{\left[\tau_{i}, \tau_{i+1}\right)}(t)
$$

where $\tau_{0}=0, \tau_{m+1}=\infty, 0<v_{0}<v_{1}<\ldots<v_{m}$, and $I_{\left[\tau_{i}, \tau_{i+1}\right)}(t)=1$ if $t \in\left[\tau_{i}, \tau_{i+1}\right)$ and 0 otherwise.

In the following, we look at the scenario when $\varphi(t)$ takes the form shown in Eq. (19).

Let $H_{0}\left(t, \tau_{k}\right)=\exp \left\{-\int_{\tau_{k}}^{t} \lambda_{\mathrm{b}}(u) d u\right\}$. Then from Eq. (1) and Eq. (19), one can obtain the reliability of the operational system as follows.

Lemma 4 The reliability distribution of the operational system is given by

$$
\bar{F}_{\mathrm{b}, 0}(t)=I_{\left[\tau_{0}, \tau_{1}\right)}(t) H_{0}\left(t, \tau_{0}\right)+\sum_{k=1}^{m}\left(I_{\left[\tau_{k}, \tau_{k+1}\right)}(t) H_{0}\left(t, \tau_{k}\right) \prod_{j=0}^{k-1} H_{0}\left(\tau_{j+1}, \tau_{j}\right)\right) .
$$


and

$$
\bar{F}_{\mathrm{o}, 0}(t)=I_{\left[\tau_{0}, \tau_{1}\right)}(t)\left(H_{0}\left(t, \tau_{0}\right)\right)^{v_{0}}+\sum_{k=1}^{m}\left(I_{\left[\tau_{k}, \tau_{k+1}\right)}(t)\left(H_{0}\left(t, \tau_{k}\right)\right)^{v_{k}} \prod_{j=0}^{k-1}\left(H_{0}\left(\tau_{j+1}, \tau_{j}\right)\right)^{v_{j}}\right) .
$$

Denote

$$
\begin{aligned}
H(t, s) & =I_{\left[\tau_{0}-s, \tau_{1}-s\right)}(t)\left(H_{0}\left(t, \tau_{0}-s\right)\right)^{v_{0}} \\
& \left.+\sum_{k=1}^{m}\left(I_{\left[\tau_{k}-s, \tau_{k+1}-s\right)}(t)\left(H_{0}\left(t, \tau_{k}-s\right)\right)^{v_{k}} \prod_{j=0}^{k-1}\left(H_{0}\left(\tau_{j+1}-s\right), \tau_{j}-s\right)\right)^{v_{j}}\right)
\end{aligned}
$$

Then we have the following Lemma.

Lemma 5 The distribution of the virtual age of the operational system is given by

$$
\bar{G}_{\mathrm{o}, n}(x)=\bar{G}_{\mathrm{o}, n-1}(x)+\int_{0}^{x} \frac{H\left(y+\frac{x-y}{\rho}, \frac{(1-\rho) y}{\rho}\right)}{H\left(y, \frac{(1-\rho) y}{\rho}\right)} d G_{\mathrm{o}, n-1}(y) .
$$

\subsubsection{Some remarks}

As reviewed above, many repair models have been proposed in the reliability literature (Nakagawa, 1988; Kijima, 1989; Doyen \& Gaudoin, 2004; Wu \& Zuo, 2010). From Section 4, it can be seen that models - such as the $\mathrm{ARI}_{m}$ and $\mathrm{ARI}_{\infty}$ in Doyen and Gaudoin (2004), or those linear maintenance models in Wu and Zuo (2010) — proposed on the basis of reduction of failure intensity can be directly used to model repairable systems with time-dependent covariates. If age-reduction models that are more complicated than ARA1 - such as the virtual age model II in Kijima (1989), $\mathrm{ARA}_{m}$ and $\mathrm{ARA}_{\infty}$ in Doyen and Gaudoin (2004), or those nonlinear maintenance models in Wu and Zuo (2010) — are applied to model (1), it is still possible to derive the lifetime distribution of the operational system after repair, or the failure process. This will be our future work.

Assumption B assumes that extrinsic factors quantified by a covariate can be maintained to the same extent as the baseline system. Assumptions $\mathrm{A}$ and $\mathrm{C}$ on the other hand do not assume this. This section briefly discusses maintenance of extrinsic factors. In some real situations, extrinsic factors represented by a covariate may be maintainable. For example, the pressure of water flowing inside a buried pipeline can be adjusted and therefore can be considered as maintainable, or the pressure from above ground may also be maintainable (on repair a pipeline may be re-routed, etc.). In the literature, however, little attention has been devoted to consider 
the effect of maintainable extrinsic factors and hence covariates on the system failure process. It should be noted, however, there is the following difference between maintenance of baseline systems and maintenance of extrinsic factors. From the perspective of reliability theory, the effect of maintenance on $\lambda_{\mathrm{o}}(t)$ or $\lambda_{\mathrm{b}}(t)$ can be estimated with observed data such as time between failures, but it cannot be measured with measurement instrument. For example, in the Kijima model (Kijima, Morimura, \& Suzuki, 1988), the virtual age can be estimated but is not measurable. In contrast, the effect of a maintenance intervention on $z(t)$ is typically measurable. For example, the change in the internal or external pressure on a buried water pipeline before and after a maintenance can be measured. That is, the extent to which maintenance (of the covariate) of the internal pressure adjusts the water pressure on the pipeline is known.

\section{A numerical example}

To validate the deviations in Section 4 , this section presents a numerical example. Suppose that $\lambda_{b}(t)=\frac{\beta}{\alpha^{\beta}} t^{\beta-1}$, where $\alpha=200$, and $\beta=1.5$. Assume that

$$
\varphi(t)= \begin{cases}2, & \text { if } t \leq 140 \\ 10, & \text { if } t>140\end{cases}
$$

Consider the scenarios under Assumptions A, B, and C, respectively. We assume the repair model is the $\mathrm{ARA}_{1}$, as shown in Eq. (12), under the three assumptions. We generate survival times up to and including the 5th repair with Monte Carlo simulation using 20,000 repetitions. The average survival interval after the $n$th repair $(n=0, . ., 4)$ are shown in Table 2 , where $n=0$ means the survival time from new. Table 2 essentially shows the average time between two adjacent repairs under the three assumptions for $\rho=0.2, \rho=0.4, \rho=0.6$ and $\rho=0.8$, respectively. For example, 180.30 in cell $(3,2)$ in Table 2 is the average time to first failure over the 20,000 simulations under Assumption A, and 97.77 in cell $(3,3)$ is the average time to first failure over the 20,000 simulations under Assumption B. The last row includes the sum of the survival intervals in the same column. For example, the value 746.33 in cell $(8,2)$ is the sum of the values $180.30,158.86,144.05,135.22$ and 127.90 in column 3 .

It can be seen that under Assumption C (for which the extrinsic factor represented by the covariate is not maintained at repair), the survival time after repair decreases dramatically in 
comparison to Assumption A. This is because under assumption A failures are not influenced by the covariate. Under Assumption B we see that the survival time after the 1st repair is longer than under assumption C. This is because under B the extrinsic factor represented by the covariate is maintained at repair. Further, the size of this difference is greater for smaller $\rho$ (greater repair effect), see figure 1. For survival times after the 2nd, 3rd repair, this difference (between A and B) is less pronounced because, by the time of the 2nd and 3rd repair, the virtual age of the system is large enough for the dramatic rise in the intensity (the intensity is 5 times larger when the virtual age exceeds 140) to have an effect even when both the extrinsic factor represented by the covariate is maintained at repair (assumption B) and $\rho$ is small. Simulation variation accounts for the differences on the first row (average survival time to first failure) within each assumption. These observations confirm that the survival times post-repair behave as we would expect.

Table 2

Average survival time following repair and average total time to failure following the 4th repair, for different values of $\rho$, the repair effect, under Assumptions A, B and C.

\begin{tabular}{|c|ccc|ccc|ccc|ccc|}
\hline & \multicolumn{4}{|c|}{$\rho=0.2$} & \multicolumn{3}{c|}{$\rho=0.4$} & \multicolumn{3}{c|}{$\rho=0.6$} & \multicolumn{3}{c|}{$\rho=0.8$} \\
\hline$n$ & $\mathrm{~A}$ & $\mathrm{~B}$ & $\mathrm{C}$ & $\mathrm{A}$ & $\mathrm{B}$ & $\mathrm{C}$ & $\mathrm{A}$ & $\mathrm{B}$ & $\mathrm{C}$ & $\mathrm{A}$ & $\mathrm{B}$ & $\mathrm{C}$ \\
\hline 0 & 180.30 & 97.77 & 97.77 & 180.31 & 97.44 & 97.44 & 179.74 & 97.52 & 97.52 & 181.25 & 98.01 & 98.01 \\
1 & 158.86 & 92.99 & 62.00 & 145.42 & 82.61 & 59.33 & 134.58 & 72.82 & 57.09 & 125.92 & 63.13 & 55.30 \\
2 & 144.05 & 26.87 & 26.98 & 127.58 & 22.87 & 22.49 & 116.44 & 20.46 & 19.86 & 106.00 & 19.03 & 17.78 \\
3 & 135.22 & 23.79 & 24.92 & 116.23 & 19.18 & 20.12 & 103.55 & 16.54 & 17.19 & 95.31 & 14.98 & 15.36 \\
4 & 127.90 & 23.09 & 24.07 & 107.63 & 18.45 & 19.26 & 95.87 & 16.03 & 16.59 & 87.40 & 14.45 & 14.76 \\
\hline Total & 746.33 & 264.51 & 235.74 & 677.17 & 240.55 & 218.64 & 630.18 & 223.37 & 208.25 & 595.88 & 209.6 & 201.21 \\
\hline
\end{tabular}

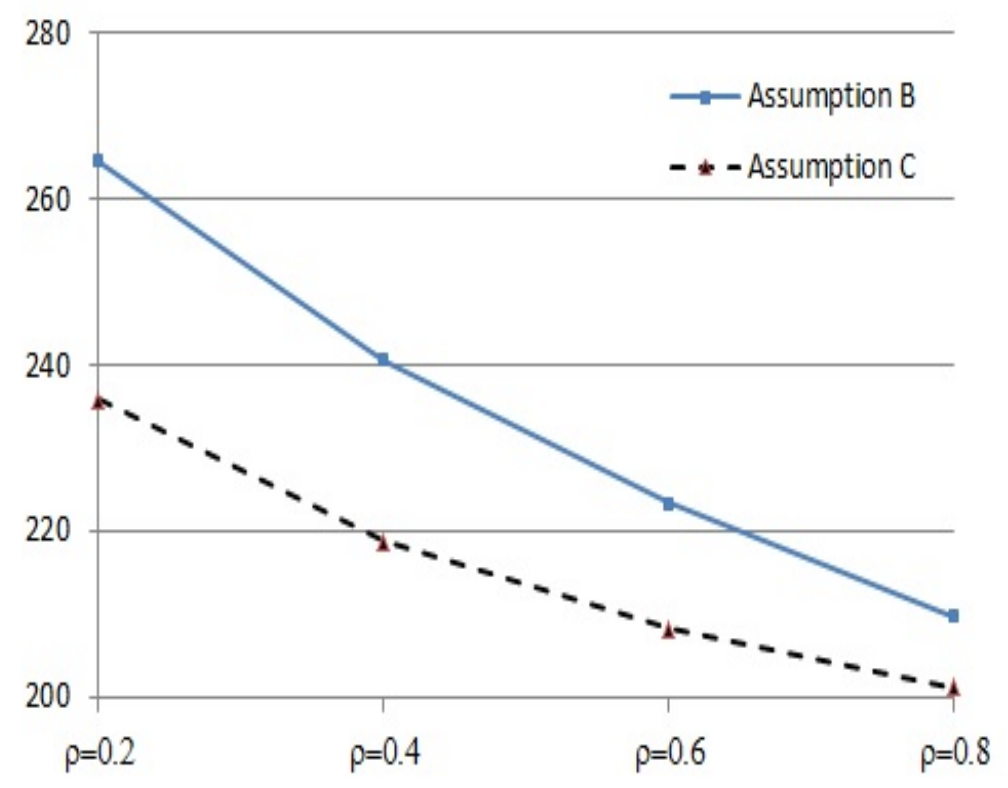

Fig. 1. Average total time to failure following the 4th repair under assumptions B (extrinsic factor maintained) and $\mathrm{C}$ (extrinsic factor not maintained) vs the size of the repair effect $\rho$. 


\section{Conclusions}

Modelling the repair effect plays an important role in optimisation of maintenance policies. The existing methods for modelling the repair effect have not considered time-dependent covariates that can influence the failure process of systems. This needs to be studied as maintaining a repairable system does not imply that extrinsic factors quantified through covariates are also maintained. For example, a maintenance action on a system may restore the system to a younger status, but it does mean that the covariates of the system will be also brought to a younger status or have the same virtual ages as the system. This causes a problem in that the argument in the baseline intensity function and the argument in the covariates are unsynchronized. Particular models such as the proportional intensity models in condition-based maintenance are becoming popular and highlighted by researchers and practitioners. It is important for maintenance policy modellers to recognise the problem of asynchronicity after repair. This problem is tackled in this paper.

This is the first paper to investigate a class of multiplicative intensity models in which maintenance intervention (service, preventive maintenance, or repair) not only modifies the baseline intensity but also the arguments of covariates. The baseline intensity quantifies intrinsic factors that affect system reliability; the covariates quantify the level or value of extrinsic factors that affect system reliability. Thus we are the first to develop models of repair in which both intrinsic and extrinsic factors are influenced by maintenance intervention and in which the extent of the influence upon intrinsic factors may be different from that upon extrinsic factors.

The findings of this paper include:

- when the effect of the covariates on maintained systems is time-independent, existing approaches to modelling the repair effect for the baseline system can be directly applied to model that for the operational system;

- when the effect of the covariates on maintained systems is time-dependent and the repair effect models are based on reduction of failure intensity, existing approaches to modelling repair effect for the baseline system can be directly applied to model that for the operational system;

- when the effect of the covariates on maintained systems is time-dependent and the repair effect models are based on reduction of age, existing approaches to modelling repair effect 
for the baseline system cannot be directly applied to model that for the operational system;

- we develop new results to consider this last case.

As we mentioned in the discussion section, our future work is to study situations when more complicated age-reduction models are applied to the baseline systems and the argument in the covariates is not synchronised with the argument in the baseline failure intensity. The difficulty here is that it is not easy to find a function that can map the argument in the baseline function to the argument in the covariates.

\section{Acknowledgements}

The authors are indebted to the reviewers and the editor for their constructive comments.

\section{References}

Andersen, P. K., \& Gill, R. D. (1982). Cox's regression model for counting processes: A large sample study. Ann.Statist., 10, 1100-1120.

Dekker, R., \& Scarf, P. (1998). On the impact of optimisation models in maintenance decision making: The state of the art. Reliability Engineering and System Safety, 60(2), 111-119.

Doyen, L., \& Gaudoin, O. (2004). Classes of imperfect repair models based on reduction of failure intensity or virtual age. Reliability Engineering and System Safety, 84(1), 45-56.

Guo, H. R., Liao, H., Zhao, W., \& Mettas, A. (2007). A new stochastic model for systems under general repairs. IEEE Transactions on Reliability, 56(1), 40-49.

Han, A., \& Hausman, J. (1990). Flexible parametric estimation of duration and competing risk models. Journal of Applied Econometrics, 5(1), 1-28.

Jiang, S., Landers, T. L., \& Rhoads, T. R. (2006). Assessment of repairable-system reliability using proportional intensity models: A review. IEEE Transactions on Reliability, 55(2), 328-336.

Kalbfleisch, J. D., \& Prentice, R. L. (2002). The Statistical Analysis of Failure Time Data (2nd ed.). John Wiley \& Sons. 
Kijima, M. (1989). Some results for repairable systems with general repair. Journal of Applied Probability, 26(1), 89-102.

Kijima, M., Morimura, H., \& Suzuki, Y. (1988). Periodical replacement problem without assuming minimal repair. European Journal of Operational Research, 37(2), 194-203.

Kirschenmann, T., Popova, E., Damien, P., \& Hanson, T. (2014). Decision dependent stochastic processes. European Journal of Operational Research, 234(3), 731 - 742.

Lam, Y. (1988). Geometric processes and replacement problem. Acta Mathematicae Applicatae Sinica, 4(4), 366-377.

Malik, M. A. K. (1979). Reliable preventive maintenance scheduling. AIIE Transactions (American Institute of Industrial Engineers), 11(3), 221-228.

Meyer, B. (1990). Unemployment insurance and unemployment spells. Econometrica, 58(4), 757-782.

Nakagawa, T. (1988). Sequential imperfect preventive maintenance policies. IEEE Transactions on Reliability, $37(3), 295-298$.

Scarf, P. (1997). On the application of mathematical models in maintenance. European Journal of Operational Research, $99(3), 493-506$.

Wang, H. (2002). A survey of maintenance policies of deteriorating systems. European Journal of Operational Research, 139(3), 469-489.

Wu, S., \& Clements-Croome, D. (2006). A novel repair model for imperfect maintenance. IMA Journal Management Mathematics, 17(3), 235-243.

Wu, S., \& Zuo, M. (2010). Linear and nonlinear preventive maintenance. IEEE Transactions on Reliability, 59(1), 242-249.

Xiang, Y. (2013). Joint optimization of x control chart and preventive maintenance policies: A discrete-time markov chain approach. European Journal of Operational Research, 229(2), 382-390.

Zhong, C., \& Jin, H. (2014). A novel optimal preventive maintenance policy for a cold standby system based on semi-markov theory. European Journal of Operational Research, 232(2), 405-411. 


\section{Appendix}

- Proof of Lemma 1. If $V_{\mathrm{o}, n-1}=y$, then from Eq. (13), the actual calendar time for which the operational system has been operated is $y / \rho$, that is, $\sum_{i=1}^{n-1} X_{\mathrm{b}, i}=y / \rho$. In other words, at the time when the system has a virtual age $y$ after the $(n-1)$ th repair, the covariates is aged $y / \rho$. Hence, we can obtain the following derivation.

$$
\begin{aligned}
\operatorname{Pr}\left\{X_{\mathrm{o}, n} \leq x \mid V_{\mathrm{o}, n-1} \geq y\right\} & \left.=\operatorname{Pr}\left\{\left(X_{\mathrm{b}, n} \leq x\right) \cap \mathscr{E} \mid V_{\mathrm{o}, n-1} \geq y\right)\right\} \\
& =\frac{\left.\operatorname{Pr}\left\{\left(X_{\mathrm{b}, n} \leq x\right) \cap \mathscr{E} \cap V_{\mathrm{o}, n-1} \geq y\right)\right\}}{\operatorname{Pr}\left\{V_{\mathrm{o}, n-1} \geq y\right\}} \\
& =\frac{\operatorname{Pr}\left\{\left(y \leq X_{\mathrm{b}, 0}<x+y\right) \cap \mathscr{E}\right\}}{\operatorname{Pr}\left\{\left(X_{\mathrm{b}, 0} \geq y\right) \cap \mathscr{E}\right\}} \\
& =\frac{\exp \left\{-\int_{0}^{y} \lambda_{\mathrm{b}}(u) \varphi\left(u+\frac{(1-\rho) y}{\rho}\right) d u\right\}-\exp \left\{-\int_{0}^{x+y} \lambda_{\mathrm{b}}(u) \varphi\left(u+\frac{(1-\rho) y}{\rho}\right) d u\right\}}{\exp \left\{-\int_{0}^{y} \lambda_{\mathrm{b}}(u) \varphi\left(u+\frac{(1-\rho) y}{\rho}\right) d u\right\}} \\
& =1-\exp \left\{-\int_{y}^{x+y} \lambda_{\mathrm{b}}(u) \varphi\left(u+\frac{(1-\rho) y}{\rho}\right) d u\right\} .
\end{aligned}
$$

In the above steps, the event that the virtual age of the operational system immediately after the $(n-1)$ th repair is $y$ is equivalent to the event that the age of the baseline system is older than $y$ and the covariate is not influenced by a repair conducted on the baseline system. That is, $\operatorname{Pr}\left\{V_{\mathrm{o}, n-1} \geq y\right\}=\operatorname{Pr}\left\{\left(X_{\mathrm{b}, 0} \geq y\right) \cap \mathscr{E}\right\}$. Also, $\left.\operatorname{Pr}\left\{\left(X_{\mathrm{b}, n} \leq x\right) \cap \mathscr{E} \cap V_{\mathrm{o}, n-1} \geq y\right)\right\}=\operatorname{Pr}\{(y \leq$ $\left.\left.X_{\mathrm{b}, 0}<x+y\right) \cap \mathscr{E}\right\}$ by definition. Thus, $\frac{\left.\operatorname{Pr}\left\{\left(X_{\mathrm{b}, n} \leq x\right) \cap \mathscr{E} \cap V_{\mathrm{o}, n-1}=y\right)\right\}}{\operatorname{Pr}\left\{V_{\mathrm{o}, n-1}=y\right\}}=\frac{\operatorname{Pr}\left\{\left(y \leq X_{\mathrm{b}, 0}<x+y\right) \cap \mathscr{E}\right\}}{\operatorname{Pr}\left\{\left(X_{\mathrm{b}, 0} \geq y\right) \cap \mathscr{E}\right\}}$, and this proves the lemma. 1.

- Proof of Lemma 2.

According to Eq. (23), one obtains

$$
\begin{aligned}
\bar{G}_{\mathrm{o}, n}(x) & =\operatorname{Pr}\left\{V_{\mathrm{o}, n}>x\right\} \\
& =\operatorname{Pr}\left\{\left(V_{\mathrm{o}, n}>x\right) \cap\left(V_{\mathrm{o}, n-1}>x\right)\right\}+\operatorname{Pr}\left\{\left(V_{\mathrm{o}, n}>x\right) \cap\left(V_{\mathrm{o}, n-1} \leq x\right)\right\} \\
& =\operatorname{Pr}\left\{V_{\mathrm{o}, n-1}>x\right\}+\operatorname{Pr}\left\{\left(V_{\mathrm{o}, n}>x\right) \cap\left(V_{\mathrm{o}, n-1} \leq x\right)\right\} \\
& \left.=\bar{G}_{\mathrm{o}, n-1}(x)+\int_{0}^{x} \operatorname{Pr}\left\{\left(V_{\mathrm{o}, n}>x\right) \cap\left(V_{\mathrm{o}, n-1} \leq x\right) \mid V_{\mathrm{o}, n-1}=y\right)\right\} d\left(\operatorname{Pr}\left\{V_{\mathrm{o}, n-1} \leq y\right\}\right) \\
& \left.=\bar{G}_{\mathrm{o}, n-1}(x)+\int_{0}^{x} \operatorname{Pr}\left\{\left(V_{\mathrm{o}, n-1}+\rho X_{\mathrm{b}, n}>x\right) \cap\left(V_{\mathrm{o}, n-1} \leq x\right) \cap \mathscr{E} \mid V_{\mathrm{o}, n-1}=y\right)\right\} d G_{\mathrm{o}, n-1}(y) \\
& \left.=\bar{G}_{\mathrm{o}, n-1}(x)+\int_{0}^{x} \operatorname{Pr}\left\{\left(X_{\mathrm{b}, n}>\frac{x-y}{\rho}\right) \cap \mathscr{E} \mid V_{\mathrm{o}, n-1}=y\right)\right\} d G_{\mathrm{o}, n-1}(y) \\
& =\bar{G}_{\mathrm{o}, n-1}(x)+\int_{0}^{x} \exp \left\{-\int_{y}^{y+\frac{x-y}{\rho}} \lambda_{\mathrm{b}}(u) \varphi\left(u+\frac{(1-\rho) y}{\rho}\right) d u\right\} d G_{\mathrm{o}, n-1}(y) .
\end{aligned}
$$

This proves Lemma 2. 
- Proof of Lemma 3.

As $\int_{y}^{y+\frac{x-y}{\rho}} \lambda_{\mathrm{b}}(u) \varphi\left(u+\frac{(1-\rho) y}{\rho}\right) d u$ is decreasing in $\rho, \exp \left\{-\int_{y}^{y+\frac{x-y}{\rho}} \lambda_{\mathrm{b}}(u) \varphi\left(u+\frac{(1-\rho) y}{\rho}\right) d u\right\}$ is increasing in $\rho$. Hence, $g_{x, \rho}(y)$ is increasing in $\rho$.

- Proof of Lemma 4.

We first establish Eq. (21). The proof of Eq. (20) is similar. From Eq. (1) and Eq. (19),

$$
\bar{F}_{\mathrm{o}, 0}(t)=\exp \left\{-\int_{0}^{t} \lambda_{\mathrm{b}}(u) \varphi(u) d u\right\}=\exp \left\{-\int_{0}^{t} \lambda_{\mathrm{b}}(u) \sum_{i=0}^{m} v_{i} I_{\left[\tau_{i}, \tau_{i+1}\right)}(u) d u\right\}
$$

If $t \in\left(\tau_{0}, \tau_{1}\right)$, then

$$
\begin{aligned}
\exp \left\{-\int_{0}^{t} \lambda_{\mathrm{b}}(u) \sum_{i=0}^{m} v_{i} I_{\left[\tau_{i}, \tau_{i+1}\right)}(u) d u\right\} & =I_{\left[\tau_{0}, \tau_{1}\right)}(t) \exp \left\{-v_{0} \int_{0}^{t} \lambda_{\mathrm{b}}(u) d u\right\} \\
& =I_{\left[\tau_{0}, \tau_{1}\right)}(t)\left(H_{0}\left(t, \tau_{0}\right)\right)^{v_{0}}
\end{aligned}
$$

If $t \in\left(\tau_{k}, \tau_{k+1}\right)$ with $k \geq 1$,

$$
\exp \left\{-\int_{0}^{t} \lambda_{\mathrm{b}}(u) \sum_{i=0}^{m} v_{i} I_{\left[\tau_{i}, \tau_{i+1}\right)}(u) d u\right\}=\sum_{k=1}^{m}\left(I_{\left[\tau_{k}, \tau_{k+1}\right)}(t)\left(H_{0}\left(t, \tau_{k}\right)\right)^{v_{k}} \prod_{j=0}^{k-1}\left(H_{0}\left(\tau_{j+1}, \tau_{j}\right)\right)^{v_{j}}\right) .
$$

Combining Eqs. (26) and (27), one can obtain Lemma 4.

- Proof of Lemma 5.

$$
\begin{aligned}
\exp \left\{-\int_{y}^{y+\frac{x-y}{\rho}} \lambda_{\mathrm{b}}(u) \varphi\left(u+\frac{(1-\rho) y}{\rho}\right) d u\right\} & =\frac{\exp \left\{-\int_{0}^{y+\frac{x-y}{\rho}} \lambda_{\mathrm{b}}(u) \varphi\left(u+\frac{(1-\rho) y}{\rho}\right) d u\right\}}{\exp \left\{-\int_{0}^{y} \lambda_{\mathrm{b}}(u) \varphi\left(u+\frac{(1-\rho) y}{\rho}\right) d u\right\}} \\
& =\frac{H\left(y+\frac{x-y}{\rho} \frac{(1-\rho) y}{\rho}\right)}{H\left(y, \frac{(1-\rho) y}{\rho}\right)} .
\end{aligned}
$$

By mimicking the proof process of Lemma 4, and substituting the above quantity into Eq. (17), one can easily obtain Lemma 5.

- Proof of Theorem 1.

From Lemma 1, the survivor function $\bar{F}_{\mathrm{o}, n}(x)$ of $X_{\mathrm{o}, n}$ is given by

$$
\bar{F}_{\mathrm{o}, n}(x)=\int_{0}^{\infty}\left(\exp \left\{-\int_{y}^{x+y} \lambda_{\mathrm{b}}(u) \varphi\left(u+\frac{(1-\rho) y}{\rho}\right) d u\right\}\right) d G_{\mathrm{o}, n-1}(y) .
$$

Hence $E\left[X_{\mathrm{o}, n}\right]=\int_{0}^{\infty} \int_{0}^{\infty}\left(\exp \left\{-\int_{y}^{x+y} \lambda_{\mathrm{b}}(u) \varphi\left(u+\frac{(1-\rho) y}{\rho}\right) d u\right\}\right) d x d G_{\mathrm{o}, n-1}(y)$.

Suppose $y_{1}>y_{2}>0$. Since both $\varphi(u)$ and $\lambda_{b}(u)$ are non-decreasing, $\lambda_{\mathrm{b}}(u) \varphi\left(u+\frac{(1-\rho) y_{1}}{\rho}\right) \geq$ $\lambda_{\mathrm{b}}(u) \varphi\left(u+\frac{(1-\rho) y_{2}}{\rho}\right)$. The interval $\left(y_{1}, x+y_{1}\right)$ has the same length as $\left(y_{2}, x+y_{2}\right)$, but $y_{1}>y_{2}$ and $y_{1}+x>y_{2}+x$, hence the area under the curve $\lambda_{\mathrm{b}}(u) \varphi\left(u+\frac{(1-\rho) y}{\rho}\right)$ within $\left(y_{1}, x+y_{1}\right)$ is greater 
than that within $\left(y_{2}, x+y_{2}\right)$. Then we have $\int_{y_{1}}^{x+y_{1}} \lambda_{\mathrm{b}}(u) \varphi\left(u+\frac{(1-\rho) y_{1}}{\rho}\right) d u \geq \int_{y_{2}}^{x+y_{2}} \lambda_{\mathrm{b}}(u) \varphi(u+$ $\left.\frac{(1-\rho) y_{2}}{\rho}\right) d u$, which implies $\int_{0}^{\infty}\left(\exp \left\{-\int_{y}^{x+y} \lambda_{\mathrm{b}}(u) \varphi\left(u+\frac{(1-\rho) y}{\rho}\right) d u\right\}\right) d x$ is decreasing in $y$. It follows that $V_{\mathrm{o}, n-1}^{A} \prec_{d} V_{\mathrm{o}, n-1}^{B}$ implies $E\left[X_{\mathrm{o}, n}^{B}\right] \leq E\left[X_{\mathrm{o}, n}^{A}\right]$, where $V_{\mathrm{o}, n-1}^{A}$ is the virtual age of the operational system immediately after the $(n-1)$ th repair and the repair effect is $A$ and a similar definition applies to $V_{\mathrm{o}, n-1}^{B}$. Thus, by definition, $E\left[S_{\mathrm{o}, n}^{B}\right] \leq E\left[S_{\mathrm{o}, n}^{A}\right]$ for all $n \geq 1$. 